\title{
Using the Support Vector Machine Algorithm to Predict $\beta$-Turn Types in Proteins
}

\author{
Xiaobo Shi, Xiuzhen Hu \\ College of Sciences Inner Mongolia University of Technology, Hohhot, China \\ Email: hh_xx_zz@yahoo.com.cn \\ Received ********* 2013
}

\begin{abstract}
The structure and function of proteins are closely related, and protein structure decides its function, therefore protein structure prediction is quite important. $\beta$-turns are important components of protein secondary structure. So development of an accurate prediction method of $\beta$-turn types is very necessary. In this paper, we used the composite vector with position conservation scoring function, increment of diversity and predictive secondary structure information as the input parameter of support vector machine algorithm for predicting the $\beta$-turn types in the database of 426 protein chains, obtained the overall prediction accuracy of 95.6\%, 97.8\%, 97.0\%, 98.9\%, 99.2\%, 91.8\%, 99.4\% and 83.9\% with the Matthews Correlation Coefficient values of $0.74,0.68,0.20,0.49,0.23,0.47,0.49$ and 0.53 for types I, II, VIII, I', II', IV, VI and nonturn respectively, which is better than other prediction.
\end{abstract}

Keywords: Support Vector Machine Algorithm; Increment of Diversity Value; Position Conservation Scoring Function Value; Secondary Structure Information

\section{Introduction}

Protein secondary structure prediction is an intermediate step in overall tertiary structure prediction. The secondary structure of a protein consists of regular, local regular and non-regular secondary structure. Local regular secondary structure contains tight turns and $\Omega$ loops. Tight turns can be divided into $\delta$-, $\gamma$-, $\beta$-, $\alpha$ - and $\pi$-turns according to the number of residues involved [1,2]. $\beta$-turns are the most common and largest number turns, which constitute about $25 \%$ of the residues in proteins [1,3-5]. $\beta$-turn is a four-residue reversal in a protein chain that the distance between the residues $\mathrm{i}$ and $i+3$ is less than $7 \AA$ and the two central residues $(i+1$ and $i+2)$ must not be helical. According to the $\psi / \varphi$ values of the central residues $i+1$ and $i+2, \beta$-turns are classified into nine types: I, II, VIII, I', II', VIa1, VIa2, VIb and IV [3-7]. Mostly, $\beta$-turn types VIa1, VIa2 and VIb are merged into one type, called type VI [3,5].

$\beta$-turn plays a vital role in protein, such as folding stability, recognition and structure assembly [2,8] and can provide templates information for drug molecule design, such as anesthetic, pesticide and antigen, etc. [1]. According to the $\psi / \varphi$ values of $\beta$-turn residues we can build up a complete three-dimensional structure for a given primary sequence. Thus, it is important to develop a method, which can predict $\beta$-turn types with high accuracy [4].
Some methods have been developed for prediction of $\beta$-turn types, such as propensities [5,6,9], sequence-coupled model [3], neural networks (NN) algorithm $[4,7,8]$ and support vector machine (SVM) algorithm [10]. In 2008, Kirschner and Frishman [7] using NN algorithm to predicting the $\beta$-turn types, obtained the best prediction performance among above works, the $Q_{\text {total }}$ for types I, II, VIII, IV, I' and II' are $85.4 \%, 96.2 \%, 93.0 \%, 85.2 \%$, $98.8 \%$ and $98.6 \%$, and the $M C C$ are $0.31,0.34,0.08$, $0.19,0.36$ and 0.14 , respectively.

In this work, we improved the input parameters of the SVM and used the seven-fold cross-validation to predicting the $\beta$-turn types in the widely used database which contained 426 protein chains, achieved better prediction result than previous studies. Furthermore, to test the effect of the database size, we also predicted $\beta$-turn types in other two databases contained 547 and 823 protein chains, respectively.

\section{Materials and Methods}

\section{1) Database}

In this paper, we used the database contained 426 protein chains, called SET426. The SET426 described by Guruprasad and Rajkumar [11] that has been widely used in $\beta$-turn type prediction [4,5,7]. And we also used other two databases contained 547 and 823 protein chains, called SET547 and SET823, respectively. The SET547 and 
SET823 described by Fuchs and Alix [5]. The databases contain chains solved by X-ray crystallography with a resolution better than $2.0 \AA$, and no two protein chains have $>25 \%$ identity. The numbers of $\beta$-turn types and nonturn are shown in Table $\mathbf{1 .}$

There are one hundred and ninety one proteins in the SET426 are contained in the SET547, ninety proteins in the SET426 are contained in the SET823 and two hundred and ten proteins in the SET547 are contained in the SET823.

\section{2) Extracted Segments}

According to the Fuchs and Alix's [5] work, the prediction of the $\beta$-turn types on a given window which contained $L$ amino acids, that the center amino acid is a $\beta$ turn (residues $i$ to $i+3$ ) with $m$ flanking residues on the left ( $i-\mathrm{m}$ to $i-1)$ and $\mathrm{n}$ flanking residues on the right $(i+4$ to $i+3+n)$. In Fuchs and Alix's [5] work, they selected the window which contained 12 amino acids. In our work, we found that the optimal window size is 10 residues long ( $m=n=3$ ). So we selected the window which contained 10 amino acids to predict the $\beta$-turn types in proteins.

3) The Position Conservation Scoring Function (DF)

The position conservation scoring function algorithm is a simple but effective forecast model. In this work, we only calculate the scores of $\beta$-turn types, the score of segment $\mathrm{S}$ can be defined as [2].

$$
\begin{gathered}
S=\sum_{i=1}^{20} C_{i}\left(p_{i j}-p_{i, \min }\right) / \sum_{i=1}^{20} C_{i}\left(p_{i, \max }-p_{i, \min }\right) \\
P_{i j}=\left(n_{i j}+\sqrt{N_{i}} / 20\right) /\left(N_{i}+\sqrt{N_{i}}\right) \\
C_{i}=100 / \log 20\left(\sum_{j=1}^{20} P_{i j} \log p_{i j}+\log 20\right)
\end{gathered}
$$

Where $j$ is the 20 amino acids, $N_{i}$ is the number of amino acids in the position $i, n_{i j}$ is the number of amino acids $j$ in the position $i . P_{i, \min }$ and $P_{i, \max }$ are the minimal and maximal values of amino acid probabilities at position $i$, respectively. $P_{i j}$ is the observed probability of amino acid $j$ at position $i, C_{i}$ is the conservation index vector at position $i$.

The frequencies of 20 amino acids at each position are selected as the basic parameters. Using the training set of

Table 1. The numbers of $\beta$-turn types and nonturn extracted from the three databases.

\begin{tabular}{ccccccccc}
\hline Type & I & I' & II & II' & VIII & IV & IV & Nonturn \\
\hline SET426 & 2457 & 302 & 924 & 168 & 672 & 2542 & 132 & 21371 \\
SET547 & 2640 & 314 & 992 & 183 & 739 & 2672 & 144 & 25279 \\
SET823 & 3808 & 500 & 1393 & 271 & 971 & 3794 & 226 & 35313 \\
\hline
\end{tabular}

seven $\beta$-turn types and nonturn, arbitrary sequence segments can obtain $8 \mathrm{DF}$ values which be calculated by (1).

4) The Increment of Diversity (ID)

The increment of diversity algorithm is essentially a measure of the composition similarity level for two systems which has been applied in the recognition of protein structural class [12] and the prediction of subcellular location of proteins [13]. In this work, we only calculate the increment of diversity values of $\beta$-turn types.

In the state space of $\mathrm{s}$ dimension, the diversity measure for diversity sources $S$ : $\left\{m_{1}, m_{2}, \ldots, m_{s}\right\}$ is defined as $[12,13]$ :

$$
D(S)=M \log M-\sum_{i} m_{i} \log m_{i}
$$

In the same state space, ID between the source of diversity $X:\left\{n_{1}, n_{2}, \ldots n_{s}\right\}$ and $Y:\left\{m_{1}, m_{2}, \ldots, m_{s}\right\}$ is defined as:

Where $N=\sum n_{i}, M=\sum m_{i}$.

The frequencies of 20 amino acids at each position are selected as the basic parameters. Construct 8 diversity sources using the training sets of seven $\beta$-turn types and nonturn, arbitrary sequence segments can obtain 8 ID values which be calculated by (5).

5) Support Vector Machine (SVM)

SVM is an extremely successful learning machine based on statistical learning theory and first proposed by Vapnik [14,15], which is a convex optimization problem, thus local optimal solution is the global optimal solution. The machine conceptually implements the following idea: input vector are non-linearly mapped to a very high-dimension feature space.

In this feature space a linear decision surface is constructed $[10,14]$. In this paper, our work is a non-linearly problem, so we only introduce the linear non-separable case.

In order to allow for training errors, "soft margin" technique was introduced, which were slack variables $\xi_{i}>0$ and the relaxed separation constraint:

$$
y_{i}\left(w \cdot x_{i}+b\right) \geq 1-\xi_{i} \quad(i=1, \ldots, N)
$$

The optimal separating hyperplane can be found by:

$$
\min \left(1 / 2|\bar{w}|^{2}+C \sum_{i=1}^{N} \xi_{i}\right)
$$

Here $C$ is a regularization parameter used to decide a trade-off between the training error and the margin.

The form of the decision function is:

$$
f(x)=\operatorname{sgn}\left(\sum_{i=1}^{N} y_{i} a_{i} \cdot K\left(x, x_{i}\right)+b\right)
$$

Here, $K\left(x_{i}, x_{j}\right)$ is the kernel function. In this paper, we select the radial basis kernel function 
$\left(K\left(x_{i}, x_{j}\right)=\exp \left(-g\left\|x_{i}-x_{j}\right\|^{2}\right)\right.$.

Figure 1 is an example of a separable problem in a two dimensional space, which comes from [14]. The support vectors, marked with grey squares, define the margin of largest separation between the two classes.

SVM has been compiled into the software packages, in this paper, we use the libsvm-2.89 software packages, which can be downloaded from

http://www.csie.ntu.edu.tw/ cjlin/libsvm. The following steps were performed to predict $\beta$-turn types: first, select the input vector of the SVM; second, inputting the vector into SVM for training, we can obtain the optimal values of parameters $C$ and $g$ are all 0.5 ; third, a classifier is constructed, and then use this classifier to predict $\beta$-turn types.

\section{6) Performance Measures}

In order to measure the performance of prediction method, the four most frequently-used parameters $[4,5,7]$ percentages of observed $\beta$-turn types that are correctly predicted $\left(Q_{o b s}\right)$, percentages of correctly predicted $\beta$-turn types $\left(Q_{\text {pred }}\right)$, the Matthews Correlation Coefficient (MCC) and the overall prediction accuracies $\left(Q_{\text {total }}\right)$ have been calculated by following equations.

$$
\begin{gathered}
Q_{\text {obs }}=a_{i} /\left(a_{i}+c_{i}\right) \times 100 \\
Q_{\text {pred }}=a_{i} /\left(a_{i}+d_{i}\right) \times 100 \\
M C C=\left(a_{i} b_{i}-c_{i} d_{i}\right) / \sqrt{\left(a_{i}+c_{i}\right)\left(a_{i}+d_{i}\right)\left(b_{i}+c_{i}\right)\left(b_{i}+d_{i}\right)} \\
Q_{\text {total }}=\left[\left(a_{i}+b_{i}\right) /\left(a_{i}+b_{i}+c_{i}+d_{i}\right)\right] \times 100
\end{gathered}
$$

Where $a_{i}$ is the number of correctly classified $\beta$-turn type $i, b_{i}$ is the number of correctly classified nonturns, $\mathrm{c}_{i}$ is the number of $\beta$-turn type $i$ incorrectly classified as nonturns or some other turn type, $d i$ is the number of nonturns incorrectly classified as $\beta$-turn type $i$.

\section{Results and Discussion}

1) Predictive Results of $\beta$-turn Types in the SET426

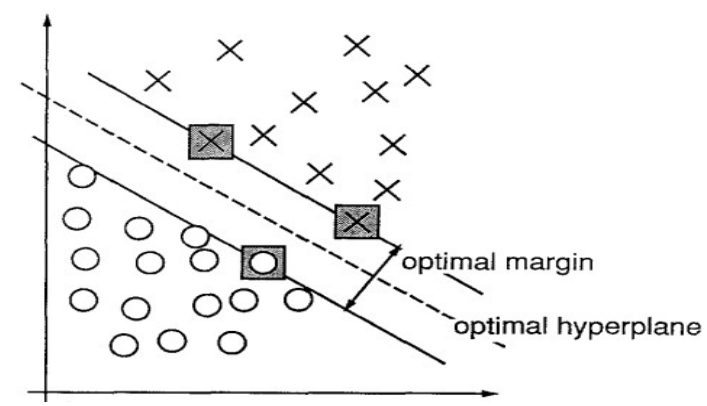

Figure 1. A separable problem in a two dimensional space.
Widely believed, the prediction performance of $\beta$-turn types can be greatly improved by using the predictive secondary structure information (PSI) $[4,5,7]$. So in this paper, we selected the PSI as input parameter of SVM. The PSI from PSIPRED [16] is encoded as follows: helix $\rightarrow$ $(1,0,0)$, strand $\rightarrow(0,1,0)$, coil $\rightarrow(0,0,1)$.

Because the prediction methods of $\beta$-turn types mostly used seven-fold cross-validation to assess the accuracy [4, 5,7], in this work we also employed seven-fold cross-validation to evaluate the performance of our method.

Using composite vector with 8 DF, 8 ID and 3 PSI as input parameter of SVM to predict the $\beta$-turn types in the SET426. The predictive result for seven-fold cross-validation is shown in Table 2. In Table 2 the MCC for every $\beta$-turn types are higher than 0.47 (except $\beta$-turn types VIII and II'). Particularly, the MCC for $\beta$-turn types I and II reach 0.74 and 0.68 respectively. The $Q_{\text {total }}$ for every $\beta$-turn types exceed $91.8 \%$. The $Q_{\text {pred }}$ for every $\beta$-turn types exceed $83.6 \%$ (except $\beta$-turn types II' and VI).

In order to comparing with other methods, the predictive result of other methods [4,5,7] for seven-fold crossvalidation in the SET426 are also shown in Table 2.

Comparing with other methods, the prediction performance of our method is better than other methods. For example, in previous work, the MCC in Kirschner's [7] work is the best among other works (except $\beta$-turn type IV), but the MCC in our method are better than Kirschner's [7] work.

2) Predictive Results of $\beta$-turn Types in the SET547 and SET823

To evaluate the predictive method, we selected the composite vector with 8 DF, 8 ID and 3 PSI as input parameter of SVM to predict the $\beta$-turn types in the SET547 and SET823, respectively. The seven-fold cross-validation results were shown in Table 3. The prediction performance in the SET823 is better than in the SET547. For example, the MCC in the SET823 is better than in the SET547 (except $\beta$-turn type I'). Compared Tables 2 and 3 , in the SET426, we obtained the best prediction performance among the three databases. The prediction results of our method in three databases are different, but the trend remained the same. The results are consistent with the Fuchs and Alix's work [5]. It denoted our method presented a strong stability whatever the database size.

\section{Conclusion}

In this work, we selected the frequencies of 20 amino acids at each position as basic parameters and in order to avoid overfitting, we used the position conservation scoring function and increment of diversity algorithms to reduce the dimension. The values of the DF, ID and PSI were used to construct the composite vector as input pa- 
Table 2. The predictive result using different methods in the SET426 using the 7-fold cross-validation.

\begin{tabular}{|c|c|c|c|c|c|c|c|c|}
\hline & Our & Kirschner's [7] & Fuchs' [5] & Kaur's [4] & Our & Kirschner's [7] & Fuchs' [5] & Kaur's [4] \\
\hline & \multicolumn{4}{|c|}{ MCC } & \multicolumn{4}{|c|}{$Q_{\text {total }}(\%)$} \\
\hline I & 0.74 & 0.31 & 0.31 & 0.29 & 95.6 & 85.4 & 84.5 & 74.5 \\
\hline I' & 0.49 & 0.36 & 0.23 & - & 98.9 & 98.8 & 94.4 & - \\
\hline II & 0.68 & 0.34 & 0.30 & 0.29 & 97.8 & 96.2 & 91.0 & 93.5 \\
\hline II' & 0.23 & 0.14 & 0.11 & - & 99.2 & 96.8 & 94.6 & - \\
\hline VIII & 0.20 & 0.08 & 0.07 & 0.02 & 97.0 & 93.0 & 90.7 & 96.5 \\
\hline IV & 0.47 & 0.19 & 0.11 & 0.23 & 91.8 & 85.2 & 84.9 & 67.9 \\
\hline VI & 0.49 & - & - & - & 99.4 & - & - & - \\
\hline \multirow[t]{2}{*}{ Nonturn } & 0.53 & - & - & - & 83.9 & - & - & - \\
\hline & \multicolumn{4}{|c|}{$Q_{o b s}(\%)$} & \multicolumn{4}{|c|}{$Q_{\text {pred }}(\%)$} \\
\hline I & 63.0 & 48.7 & 50.0 & 74.1 & 92.4 & 31.7 & 30.8 & 22.1 \\
\hline I' & 27.9 & 21.9 & 51.8 & - & 85.7 & 59.3 & 11.6 & - \\
\hline II & 52.3 & 25.2 & 52.8 & 52.8 & 92.0 & 50.2 & 22.2 & 25.5 \\
\hline II' & 38.3 & 16.3 & 32.8 & - & 66.7 & 12.7 & 4.6 & - \\
\hline VIII & 24.2 & 19.0 & 18.7 & 2.8 & 98.9 & 8.0 & 6.0 & 7.2 \\
\hline IV & 29.5 & 29.3 & 17.7 & 72.0 & 83.6 & 26.0 & 20.7 & 18.6 \\
\hline VI & 42.1 & - & - & - & 57.1 & - & - & - \\
\hline Nonturn & 98.2 & - & - & - & 83.2 & - & - & - \\
\hline
\end{tabular}

Table 3. The predictive results in the SET547 and SET823 for 7 -fold cross-validation.

\begin{tabular}{ccccc}
\hline \multirow{2}{*}{ Type } & \multicolumn{2}{c}{ SET547 } & SET823 & \multicolumn{2}{c}{ SET547 } & SET823 \\
\cline { 2 - 5 } & \multicolumn{2}{c}{ MCC } & \multicolumn{2}{c}{$\mathbf{Q}_{\text {total }}$ (\%) } \\
\hline I & 0.51 & 0.63 & 93.0 & 94.2 \\
I' & 0.53 & 0.48 & 99.0 & 98.9 \\
II & 0.55 & 0.63 & 97.3 & 97.6 \\
II' & 0.34 & 0.37 & 99.3 & 99.3 \\
VIII & 0.09 & 0.11 & 97.0 & 97.3 \\
IV & 0.29 & 0.30 & 91.1 & 91.0 \\
IV & 0.35 & 0.48 & 99.4 & 99.5 \\
Nonturn & 0.36 & 0.43 & 80.6 & 82.0 \\
\hline & \multicolumn{2}{c}{$\mathbf{Q}_{\text {obs }}$ (\%) } & \multicolumn{2}{c}{$\mathbf{Q}_{\text {pred }}(\%)$} \\
\hline I & 37.7 & 53.9 & 78.0 & 80.7 \\
I' & 37.5 & 29.6 & 75.0 & 77.8 \\
II & 42.3 & 47.7 & 76.0 & 84.8 \\
II' & 23.1 & 18.0 & 50.0 & 77.8 \\
VIII & 17.0 & 22.0 & 56.9 & 60.0 \\
IV & 14.4 & 15.5 & 71.4 & 70.6 \\
IV & 25.0 & 33.3 & 50.0 & 68.8 \\
Nonturn & 97.2 & 97.3 & 81.2 & 82.3 \\
\hline
\end{tabular}

rameter of SVM to predict the $\beta$-turn types in the SET426, the predictive results were better than the previous methods. In addition, we predicted the $\beta$-turn types in SET547 and SET823 respectively, better results were also obtained.

\section{Acknowledgements}

This work was supported by National Natural Science Foundation of China (30960090), the Natural Science Foundation of the Inner Mongolia of China (project No.2009MS0111) and Project for university of Inner Mongolia of China (project, NJZY08059).

\section{REFERENCES}

[1] K. C. Chou, "Prediction of Tight Turns and Their Types in Proteins,” Analytical Biochemistry, Vol. 286, 2000, pp. 1-16. http://dx.doi.org/10.1006/abio.2000.4757

[2] X. Z. Hu and Q. Z. Li, "Using Support Vector Machine to Predict $\beta$ - and $\gamma$-Turns and in Proteins," Journal of Computational Chemistry, Vol. 10, 2008, pp. 1-9.

[3] K. C. Chou and J. R. Blinn, "Classification and Prediction of Beta-turn Types,” Journal of Protein Chemistry, Vol. 16, 1997, pp. 575-595. http://dx.doi.org/10.1023/A:1026366706677

[4] K. S. Kaur and G. P. Raghava, “A Neural Network Method for Prediction of Beta-Turn Types in Proteins using Evolutionary Information,” Bioinformatics, Vol. 16, 2004, pp. 2751-2758.

http://dx.doi.org/10.1093/bioinformatics/bth322

[5] P. F. J. Fuchs and A. J. P. Alix, "High Accuracy Prediction of $\beta$-Turn and Their Types Using Propensities and Multiple Alignments,” Proteins, Vol. 59, 2005, pp. 828839. http://dx.doi.org/10.1002/prot.20461

[6] E. G. Hutchinson and J. M. Thornton, "A Revised Set of Potentials for Beta-turn Formation in Proteins," Protein Science, Vol. 3, 1994, pp. 2207-2216. 
http://dx.doi.org/10.1002/pro.5560031206

[7] A. Kirschner and D. Frishman, "Prediction of $\beta$-Turns and $\beta$-Turn Types by a Novel Bidirectional Elman-Type Recurrent Neural Network with Multiple Output Layers,” Gene, Vol. 422, 2008, pp. 22-29. http://dx.doi.org/10.1016/j.gene.2008.06.008

[8] A. J. Shepherd, D. Gorse and J. M. Thornton, "Prediction of the Location and Type of Beta-turns in Proteins using Neural Networks," Protein Science, Vol. 8, 1999, pp. 1045-1055. http://dx.doi.org/10.1110/ps.8.5.1045

[9] C. M. Wilmot and J. M. Thornton, "Analysis and Prediction of the Different Types of Beta-turn in Proteins," Journal of Molecular Biology, Vol. 203, 1988, pp. 221232. http://dx.doi.org/10.1016/0022-2836(88)90103-9

[10] Y. D. Cai, X. J. Liu, Y. X. Li, X. B. Xu and K. C. Chou, "Support Vector Machines for the Classification and Prediction of Beta-Turn Types,” Journal of Peptide Science, Vol. 8, 2002, pp. 297-301. http://dx.doi.org/10.1002/psc.401

[11] K. Guruprasad and S. Rajkumar, "Beta-and Gamma-Turns in Proteins Revisited: A New Set of Amino Acid TurnType Dependent Positional Preferences and Potentials," Journal of Bioscience, Vol. 25, 2000, pp. 143-156.

[12] Q. Z. Li and Z. Q. Lu, "The Prediction of the Structural Class of Protein: Application of the Measure of Diversity,” Journal of Theoretical Biology, Vol. 213, 2001, pp. 493502. http://dx.doi.org/10.1006/jtbi.2001.2441

[13] Y. L. Chen and Q. Z. Li, "Prediction of the Subcellular Location of Apoptosis Proteins," Journal of Theoretical Biology, Vol. 245, 2007, pp. 775-783. http://dx.doi.org/10.1016/j.jtbi.2006.11.010

[14] C. Cortes and V. Vapnik, "Support Vector Network," Machine Learning, Vol. 20, 1995, pp. 273-293. http://dx.doi.org/10.1007/BF00994018

[15] V. Vapnik, “Statistical Learning Theory,” Wiley-InterScience, New York, 1998.

[16] D. T. Jones, "Protein Secondary Structure Prediction Based on Position-Speck Scoring Matrices,” Journal of Molecular Biology, Vol. 292, 1999, pp. 195-202. http://dx.doi.org/10.1006/jmbi.1999.3091 